\title{
Article \\ High-Efficiency Quantum Dot Lasers as Comb Sources for DWDM Applications
}

\author{
Mario Dumont ${ }^{1, *(1)}$, Songtao Liu ${ }^{2}$, M. J. Kennedy ${ }^{1}$ and John Bowers ${ }^{1, *(\mathbb{C})}$ \\ 1 Department of Electrical and Computer Engineering, University of California, Santa Barbara, CA 93106, USA; \\ mj@engineering.ucsb.edu \\ 2 Intel Corporation, 2200 Mission College Blvd., Santa Clara, CA 95054, USA; stliu.photonics@gmail.com \\ * Correspondence: MarioDumont@ucsb.edu (M.D.); JBowers@ucsb.edu (J.B.)
}

check for updates

Citation: Dumont, M.; Liu, S.;

Kennedy, M.J.; Bowers, J.

High-Efficiency Quantum Dot Lasers

as Comb Sources for DWDM

Applications. Appl. Sci. 2022, 12, 1836.

https://doi.org/10.3390/

app12041836

Academic Editor: Edik U. Rafailov

Received: 5 January 2022

Accepted: 26 January 2022

Published: 10 February 2022

Publisher's Note: MDPI stays neutral with regard to jurisdictional claims in published maps and institutional affiliations.

Copyright: (c) 2022 by the authors. Licensee MDPI, Basel, Switzerland. This article is an open access article distributed under the terms and conditions of the Creative Commons Attribution (CC BY) license (https:// creativecommons.org/licenses/by/ $4.0 /)$.

\begin{abstract}
The trend of data center transceivers is to increase bandwidth while simultaneously decreasing their size, power consumption, and cost. While data center links have previously relied on vertical-cavity surface-emitting lasers (VCSELs) or in-plane lasers using coarse wavelength division multiplexing (WDM) to encode data, recently, dense WDM (DWDM) has moved to the forefront for next-generation links. Several approaches exist as light sources for DWDM links; DFB arrays, nonlinear microcombs, and semiconductor mode-locked lasers (MLLs). This paper focuses on quantum dot MLLs (QDMLLs), which currently offer the best efficiency, simplicity, and size. The efficiency of optical combs generated by QDMLLs is analyzed in depth in this study.
\end{abstract}

Keywords: semiconductor laser; mode locking; data centers; DWDM

\section{Introduction}

Cloud-based computing continues to fuel the demand for more internet protocol traffic, thrusting hyperscale data centers into the "Zeta-byte era" [1]. To reduce power spent on input-output (IO) and to eliminate the IO bottleneck at the front panel of server rack units [2], data center links will see photonics move into electronics packages in the near future $[3,4]$. This seriously constrains the power consumption, footprint, and spectral efficiency of next-generation links. Significant breakthroughs in silicon photonics have led to compact, power-efficient transceivers, which benefit from the high-volume production capability of silicon [5].

One technology in particular, micro-ring modulators (MRM), has allowed silicon photonic transceivers to produce optical links with extremely high IO bandwidth per $\mathrm{mm}^{2}$ [6]. MRMs benefit from a compact footprint and are resonant structures, allowing them to modulate a single wavelength in a dense wavelength division multiplexing (DWDM) signal, thus eliminating optical multiplexers and demultiplexers (MUX/DEMUX) [7]. This capability has dramatically increased the need for a monolithic light source that can deliver all DWDM channels. The three approaches typically considered to meet this need are distributed feedback laser (DFB) arrays, nonlinear optical frequency combs (NOFC), and semiconductor mode-locked lasers(MLLs) [8].

\subsection{DFB Arrays}

The archetype wavelength division multiplexing (WDM) light source, DFB arrays, are implemented in long haul, metro, and data center applications [9]. DFBs are commercially available across the entire telecom range, with wavelengths available anywhere between 760 and 14,000 $\mathrm{nm}$ [10]. The WDM source is formed by combining the optical outputs of many individual lasers. DFB arrays have two aspects that NOFCs and MLLs do not; namely, the ability to scale the power in each channel independently to the desired level, and showing no reduction in efficiency as the number of channels increases. They do, however, have the largest footprint, and require an MUX to combine individual channels into one 
fiber. Additionally, the large temperature variation inside data center racks requires control electronics to keep each individual laser aligned to the DWDM grid/MUX, increasing their energy cost per bit [11].

\subsection{Nonlinear Optical Frequency Combs}

As an alternative to DFB arrays, NOFC offer the ability to generate a spectrum of discrete optical frequencies separated by a fixed channel spacing. NOFCs typically employ micro-disk or micro-ring resonators, but a straight waveguide can be used in combination with an MLL [8]. They can offer extremely large bandwidth [12], the channel spacing can be adjusted over an extremely large range [13,14], and individual comb lines can offer superior optical linewidths, making them suitable for coherent communication [15]. Anomalous group velocity dispersion (GVD) Kerr solitons suffer from an inherently low efficiency [16]. Recently, normal GVD dark soliton combs have reached $41 \%$ on-chip pump-to-comb efficiency [17]. Their biggest drawback is that the power of individual lines is typically low [18], with an average power per line of about $-10 \mathrm{dBm}$. The comb generated usually shows large power variation across the comb (10-20 dB); however, recent improvements have increased the power per line [17]. They also suffer from an inherently low efficiency when considering net wall plug efficiency (WPE) of the entire source. Often neglected from the calculation, the wall plug efficiency of the pump laser itself must be considered. Additionally, optical coupling losses between the laser and the NOFC further decrease the efficiency; however, integration will alleviate this [19]. Excluding pump generation and coupling, conversion efficiencies can be as high as $40-60 \%[17,20]$.

\subsection{Semiconductor MLLS}

Semiconductor MLLs output a high frequency pulse train with fixed frequency-spacing between the longitudinal cavity modes in a compact, efficient, reliable, and robust device, making them a natural candidate for optical sampling [21], clock distribution [22], and as DWDM sources [23]. Quantum well MLLs have been investigated for more than three decades; however, the introduction of InAs quantum dot (QD) active region has improved their performance. QDs have been shown to have higher gain [24], a lower threshold [25], a smaller linewidth enhancement factor [26], they benefit from inhomogeneous broadening [27], improved temperature-induced threshold increase $\left(\mathrm{T}_{0}\right)$ [28], and improved carrier dynamics [29]. Implementing InAs QDs has led to the development of efficient high-output-power MLLs, which are suitable for comb sources [8]. They output dozens of comb lines, resulting in multi-terabit single device data transmission capacities [23]. Additionally, these devices do not require initiation routines or feedback loops, and they only use voltage and current sources.

\section{Device Design and Fabrication}

Currently, DWDM networks typically operate at 50 or $100 \mathrm{GHz}$ channel spacing and the lasers presented in this paper were designed for $60 \mathrm{GHz}$ operation. When channel spacing exceeds 30-40 GHz, the cavity length becomes prohibitively short and the laser cannot emit sufficient light output from passive mode locking. In order to achieve $60 \mathrm{GHz}$ spacing, colliding pulse mode locking (CPM) was employed to raise the channel spacing of lasers to multiples of the cavity FSR [30]. As an alternative to CPM, single section mode locking can also be used to achieve higher channel spacing [31]. Figure 1a shows the principle behind a colliding pulse mode-locked laser (CPMLL). The number of pulses circulating determines the harmonic (i.e., 3rd, 4th, or 5th), which is 3rd in Figure 1a. The pulses collide at interval distances of the cavity length divided by the harmonic from the output facets and saturable absorbers (SAs) are placed at these locations. For stability, the SAs closest to the two output facets must be included. The SAs at other positions in the 4th and 5th harmonic lasers can be excluded. The goal of CPM is the increase of the channel spacing of MLLs. However, the fundamental modes, which are spaced by a single FSR, are 
still present. These modes are considerably suppressed, as can be seen in Figure 1b. A side mode suppression ratio (SMSR) of $25 \mathrm{~dB}$ or greater can be achieved for optimal states.

(a)

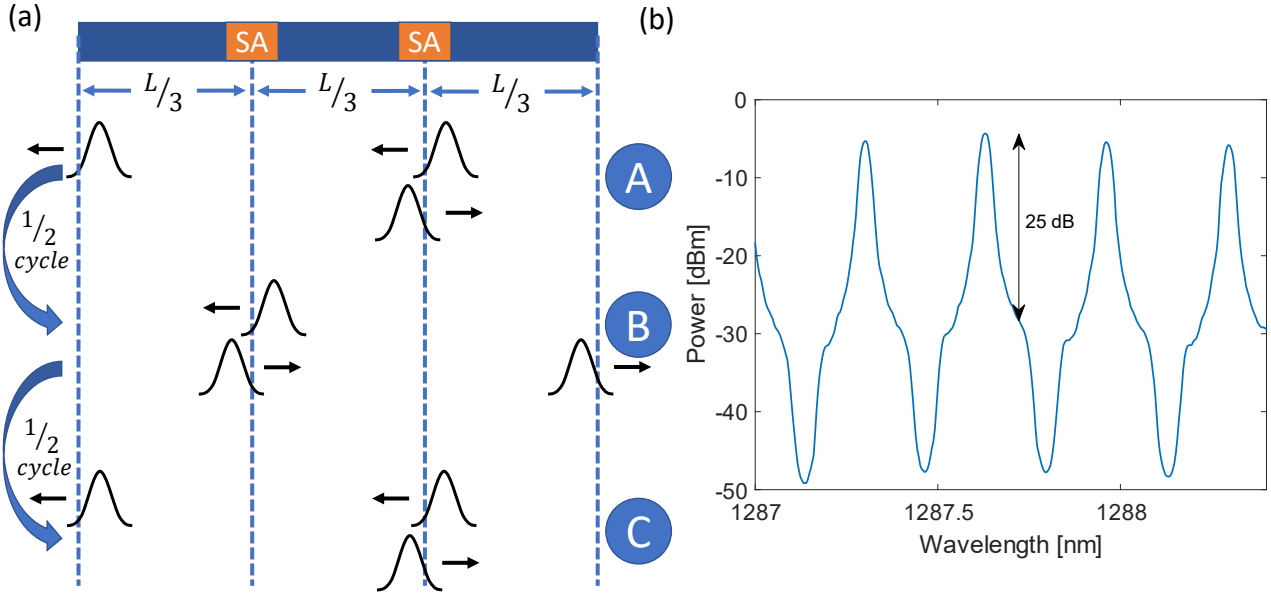

Figure 1. (a) Diagram of multiple pulses circulating in a 3rd harmonic colliding pulse mode-locked laser (CPMLL). A pulse is emitted from a facet per cycle. In position A, two pulses are colliding in the right saturable absorber (SA) and a pulse is being emitted from the left facet. The position of the three pulses half a cycle later is shown in position B. Two pulses are colliding in the left SA and a pulse is being emitted from the right facet. In position $\mathrm{C}$, one full cycle has been completed and the position of the pulses are analogous to position A. (b) enlarged optical spectrum of a 3rd harmonic CPMLL.

All devices discussed were grown on nominal (001) n-doped GaAs wafers with a VEECO GenIII solid source Molecular Beam Epitaxy machine. The details of the epi-stack are shown in Figure 2a. The stack begins with a $400 \mathrm{~nm}$ thick, highly doped n-contact layer. The aluminum content is then graded to $40 \%$ to form the waveguide lower cladding, which is $1.4 \mu \mathrm{m}$ thick. The $\mathrm{Al}$ content is then graded to $20 \%$ for a $20 \mathrm{~nm}$ thick confinement layer, before the active region. The active region is $285 \mathrm{~nm}$ thick and contains 5 layers of (quantum) dots in a well (DWELL). The DWELL layer consists of 2.5 monolayers of InAs in a $7 \mathrm{~nm}$ thick $\operatorname{In}_{.15} \mathrm{Ga}_{.85}$ As well and are separated by $37.5 \mathrm{~nm}$ of GaAs. The p-type cladding consists of the same Al concentrations and thicknesses, with a $300 \mathrm{~nm}$ thick p-type, highly doped contact layer on top. The photoluminescence of the QDs is shown in Figure 2b, and has a full width at half maximum of $38 \mathrm{meV}$.

(a) $\quad \mathrm{p}+$ GaAs contact layer $(300 \mathrm{~nm})$

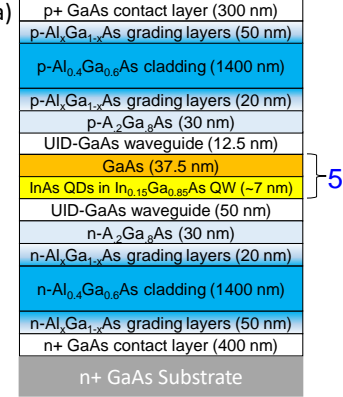

(b)

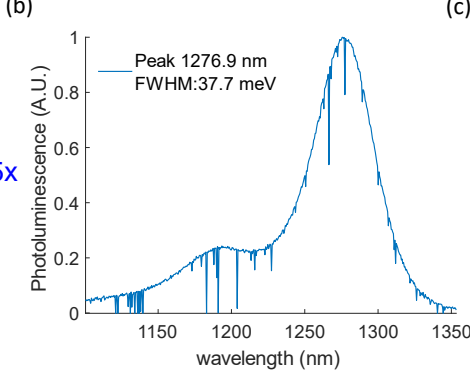

(c)

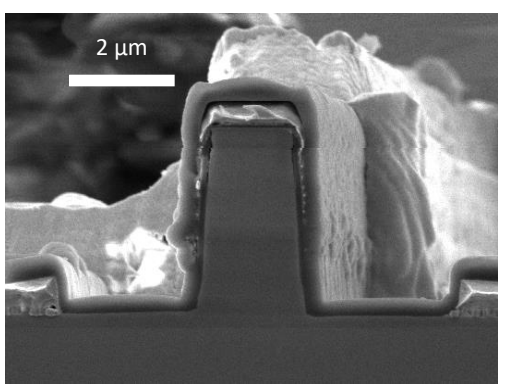

Figure 2. (a) Schematic and (b) photoluminescence of the epitaxial stack. (c) Scanning electron microscope image of a fabricated laser.

The epi-stack was processed into deeply etched ridge lasers, as seen in Figure 2c. The ridge waveguides were formed by inductively coupled plasma etching using $\mathrm{N}_{2}$ and $\mathrm{Cl}_{2}$. The etching conditions were optimized to give vertical sidewalls and reduced sidewall roughness to minimize internal loss [32]. An additional dry etch was preformed to electrically isolate the p-contact of the SA and gain section. This was accomplished by etching a $10 \mu \mathrm{m}$ long section of the ridge through the $300 \mathrm{~nm}$ p-contact layer and through $300 \mathrm{~nm}$ 
of the p-cladding. The sidewalls were first passivated with $12 \mathrm{~nm}$ of atomic layer deposition alumina to reduce sidewall recombination, followed by $1 \mu \mathrm{m}$ of sputtered $\mathrm{SiO}_{2}$. The contacts to the p-type and n-type layers were made by $\mathrm{Pd} / \mathrm{Ti} / \mathrm{Pd} / \mathrm{Au}$ and $\mathrm{Pd} / \mathrm{Ge} / \mathrm{Pd} / \mathrm{Au}$ layers to form ohmic contacts with contact resistances of about $2 \times 10^{-5} \Omega \cdot \mathrm{cm}^{2}$. The substrate was thinned to $200 \mu \mathrm{m}$ before cleaving to form output facets. All sample facets were left as-cleaved.

\section{Performance of CPMLLs}

\subsection{Performance}

Devices were tested continuous-wave on a temperature-controlled copper heatsink at $20^{\circ} \mathrm{C}$. An integration sphere was used to measure light output, and voltage was measured in a four-wire configuration. The light-current and single-sided WPE curves for the $60 \mathrm{GHz}$ 3rd harmonic CPMLLs are shown in Figure 3a,b. The total SA length, as a fraction of the cavity length $(2048 \mu \mathrm{m})$, is labeled on the respective curves. The performance of the devices are shown under two conditions; the first is with the SA sections connected in parallel with the gain section (labeled forward-biased), and the second is with the SA sections reverse-biased at $2 \mathrm{~V}$ (labeled $2 \mathrm{~V}$ ). The forward-biased performance mimics the expected performance of Fabry-Perot lasers of the same design (i.e., lasers with no SA section). There was no significant difference in the light output or voltage between the three devices. When the SA was reverse-biased, the decrease in output power due to the SA was clear. The small penalty in efficiency imposed by the SA created equal frequency spacing between comb lines. The decrease in output power as a percentage of forward-biased power is shown in Figure 3c. When considering devices with shorter SA sections, which will later be shown to be optimal for comb generation, the decrease in output power and WPE is only in the $10-20 \%$ range.
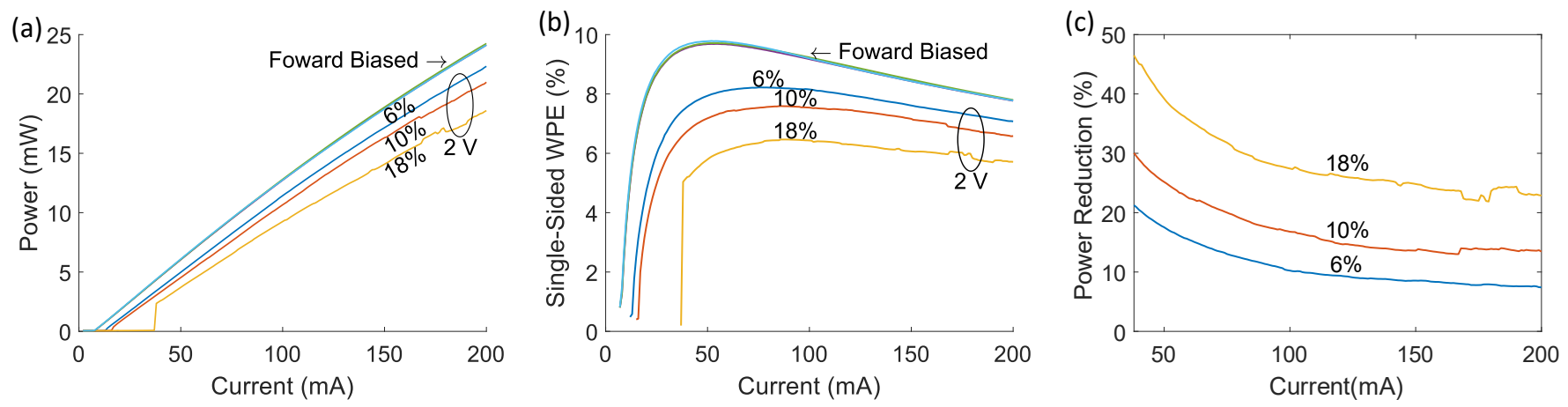

Figure 3. Comparison of 3rd harmonic quantum dot colliding pulse mode-locked lasers (CPMLLs). (a) Light-current and (b) single-sided wall plug efficiency curves with saturable absorber (SA), biased in parallel with the gain section (forward-biased), and reverse-biased at $2 \mathrm{~V}$ (circled curves). The three forward-biased curves in (a,b) are hard to distinguish, because the performance is similar. (c) The percent reduction in power from reverse biasing the SA. SA lengths are labeled on the curves as a total fraction of the cavity length.

\subsection{Comb Bandwidth}

In order to investigate the performance of the CPMLLs as optical frequency comb sources, optical spectra from the devices in Figure 3 were collected for varying SA reverse biases and gain section drive currents. To prevent laser damage, the states in the gray region in the upper right hand corner of Figure $4 \mathrm{a}-\mathrm{i}$ were not tested. These states exceeded the maximum allowed SA current, which was conservatively set at $16 \mathrm{~mA}$ total. The spectra collected were filtered by removing states with less than $15 \mathrm{~dB}$ SMSR. For all remaining states, the number of $60 \mathrm{GHz}$ channels in a $6 \mathrm{~dB}$ bandwidth were tabulated, and the results are shown in Figure $4 \mathrm{a}-\mathrm{c}$. The maximum and average bandwidths of the three devices are listed in Table 1 . The 10\% device shows the largest bandwidth, the highest average 
bandwidth, and the largest number of states with SMSR exceeding $15 \mathrm{~dB}$. Figure 5 a shows the comb with the largest achievable bandwidth of 42 comb lines, or $14.1 \mathrm{~nm}$, from the $10 \%$ device at a gain current of $200 \mathrm{~mA}$ and an SA reverse bias of $3.3 \mathrm{~V}$.

Table 1. List of important parameters from the devices tested. Saturable absorber length, as a total fraction of the cavity length, is listed as percentage in column one. The number of states measured with a side mode suppression ratio greater than $15 \mathrm{~dB}$ (SMSR $\geq 15 \mathrm{~dB}$ ) are listed in column two. The number of comb lines in the state with the largest $6 \mathrm{~dB}$ bandwidth is listed in column three. The average bandwidth of all states with SMSR $\geq 15 \mathrm{~dB}$ is listed in column four. The power of the lowest line (PoLL) of a contiguous 20 line comb is listed in column five in $\mathrm{dBm}$. The number of states with a PoLL $\geq-8 \mathrm{dBm}$ is listed in column six. The maximum wall plug efficiency of the lowest line (WPE20) is listed in column seven in dB. The average WPE20 of all states with SMSR $\geq 15 \mathrm{~dB}$ is listed in column eight in $\mathrm{dB}$.

\begin{tabular}{|c|c|c|c|c|c|c|c|}
\hline SA Length & SMSR $\geq 15 \mathrm{~dB}$ & Max. 6 dB BW & Ave. $6 \mathrm{~dB}$ BW & Max. PoLL & PoLL $\geq-8 \mathrm{dBm}$ & Max. Eff. & Ave. Eff. \\
\hline $6 \%$ & 252 & 30 & 20.1 & -6.1 & 27 & -29.2 & -32.3 \\
\hline $10 \%$ & 390 & 42 & 24.0 & -5.1 & 117 & -28.9 & -32.3 \\
\hline $18 \%$ & 304 & 35 & 18.0 & -5.2 & 51 & -29.3 & -35.3 \\
\hline
\end{tabular}

(a)

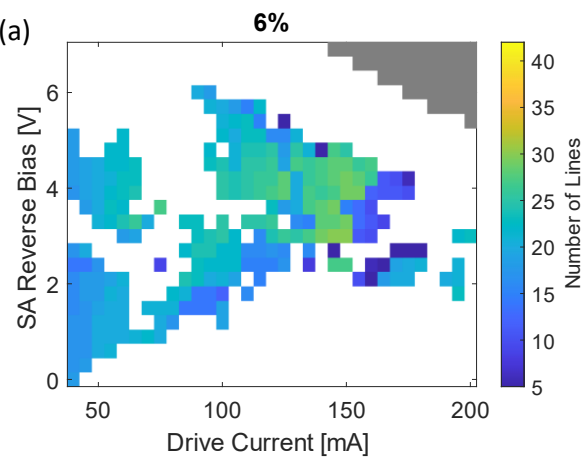

(b)

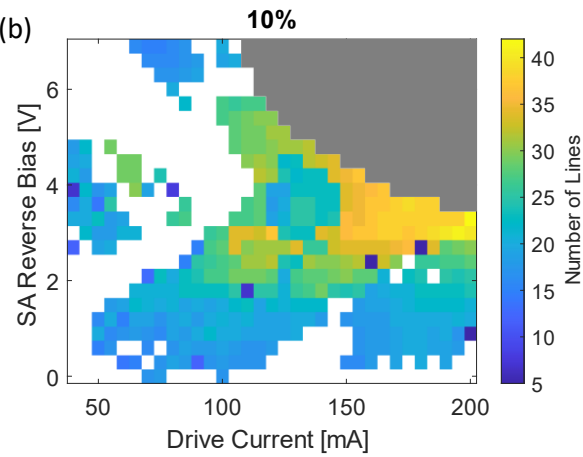

(c)

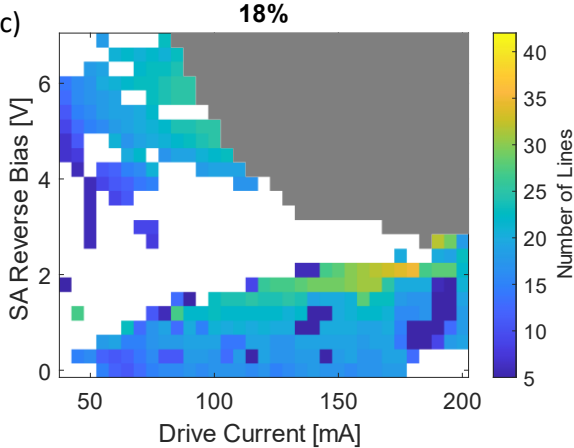

(d)

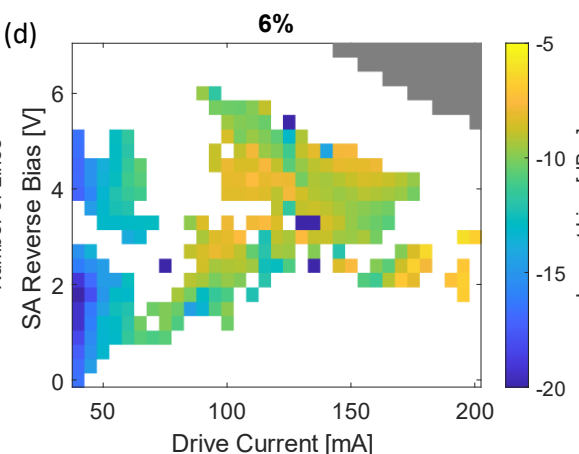

(e)

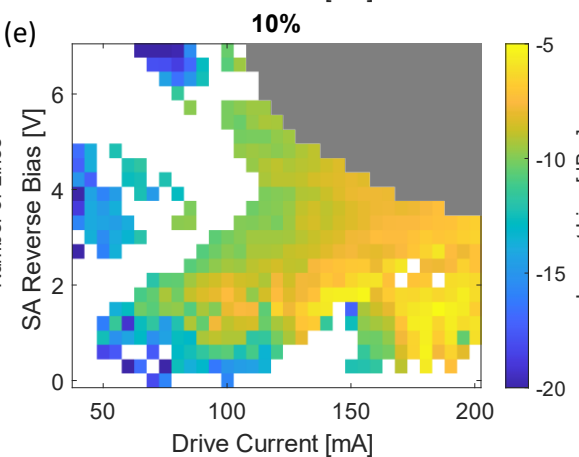

$18 \%$

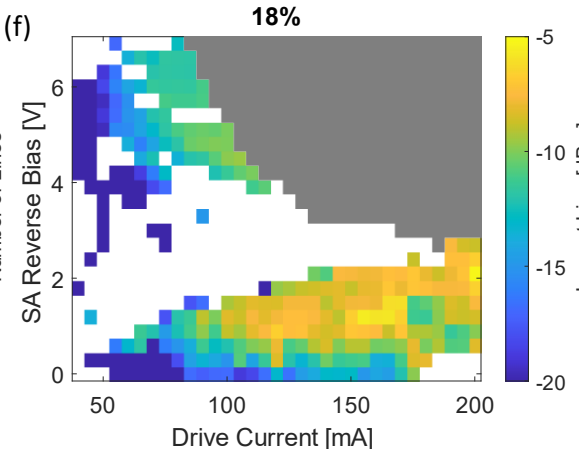

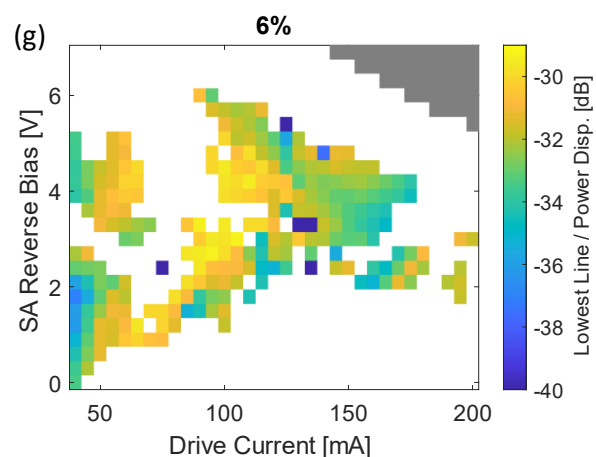

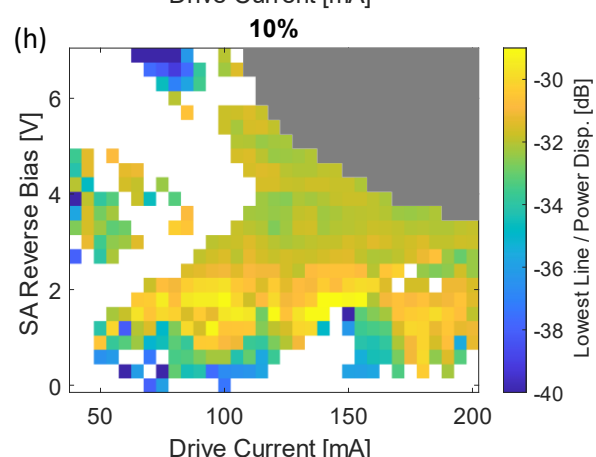

$18 \%$

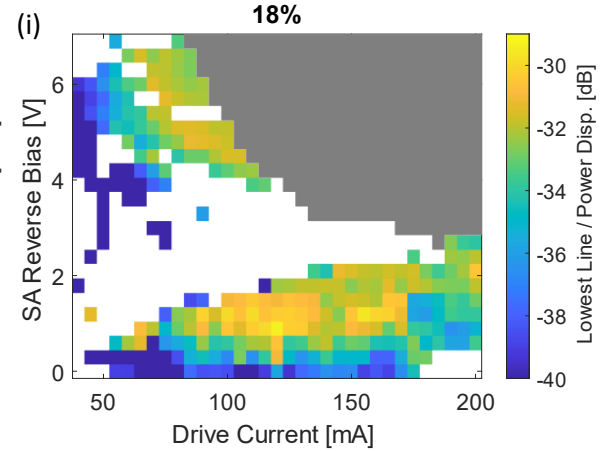

Figure 4. Color-coded plot of the $6 \mathrm{db}$ optical bandwidth $(\mathrm{a}-\mathrm{c})$, measured in the number of $60 \mathrm{GHz}$ spaced comb lines, power of the lowest line of a contiguous 20-line comb (d-f), and the wall plug efficiency of the lowest comb line $(\mathrm{g}-\mathbf{i})$ for total saturable absorb lengths of $6 \%, 10 \%$, and $18 \%$ of the cavity length. Gray regions were not tested to avoid risk of device damage. 


\subsection{Power per Line}

Of equal importance was the analysis of the power of individual comb lines of CPMLLs. Uneven power distribution between comb lines is often a limiting factor of OFCs. The optical SNR of an encoded DWM channel is, in general, proportional to the optical power. In extreme cases, comb lines with the lowest power will not be encoded with data, reducing the bandwidth and spectral efficiency of the link. To address this concern, each measured spectrum is analyzed to identify the 20 contiguous comb lines with the highest power. The power of the lowest line (PoLL) is found for each spectrum and the PoLL vs. SA bias and drive current is plotted in Figure $4 \mathrm{~d}-\mathrm{f}$. The maximum recorded PoLL and the number of states with PoLL $\geq-8 \mathrm{dBm}$ are recorded in Table 1 . For lower bias values (50-120 mA), similar PoLL values are recorded for the $6 \%$ and $10 \%$ devices. Similarly, for higher biases, the $10 \%$ and $18 \%$ devices show similar PoLL values. The 10\% device shows the highest PoLL value of $-5.1 \mathrm{dBm}$ and the most states (117) with PoLL $\geq-8 \mathrm{dBm}$. The PoLL values reported in Figure 4 and Table 1 are single-sided fiber-coupled power values. Devices have as-cleaved facets, with equal amounts of power emitted from both sides. This, in combination with the $2 \mathrm{~dB}$ fiber coupling loss, corresponds to a roughly $5 \mathrm{~dB}$ decrease in the reported values from the total power emitted from the device. This shows that with a high reflection coating and improved fiber coupling and device design, combs with PoLL $\geq 0 \mathrm{dBm}$ are achievable. The spectrum with the highest PoLL is shown in Figure $5 \mathrm{~b}$ from the $10 \%$ device at a $185 \mathrm{~mA}$ drive current and with a $1.5 \mathrm{~V}$ SA bias.

(a)
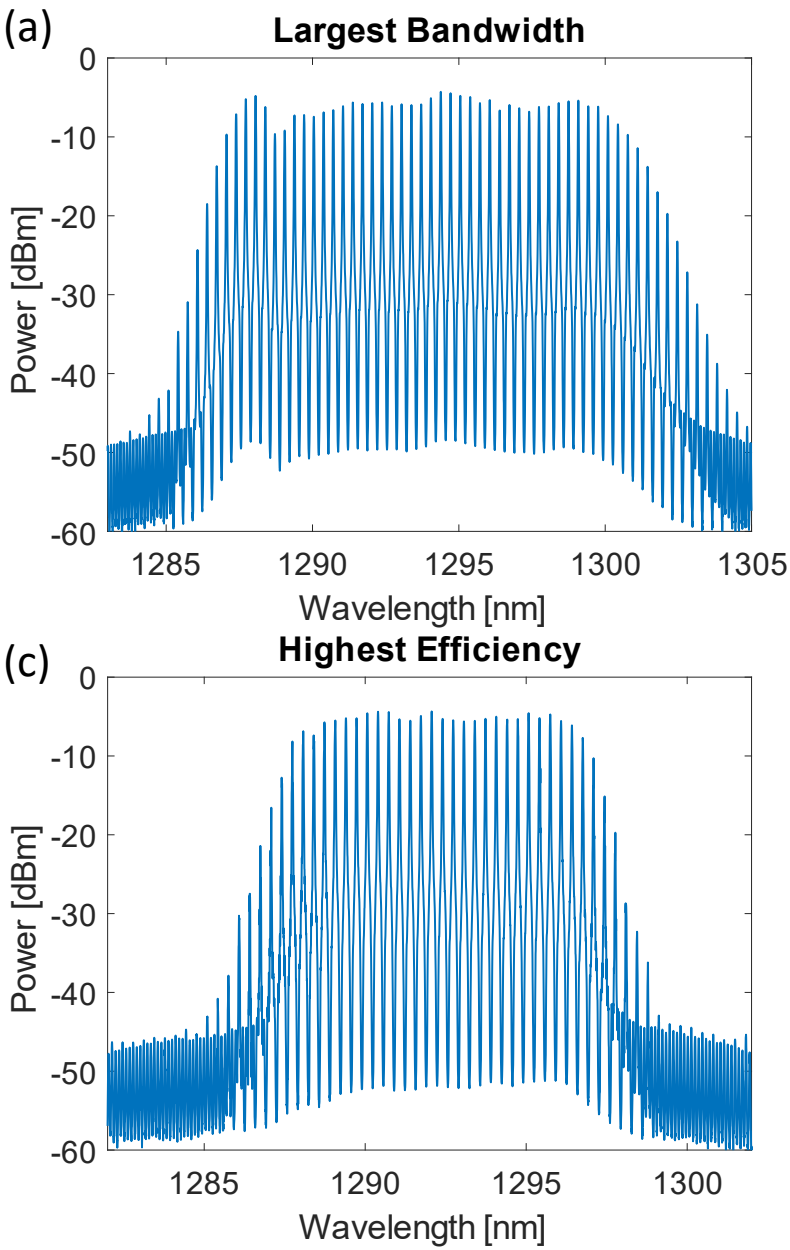

(b)

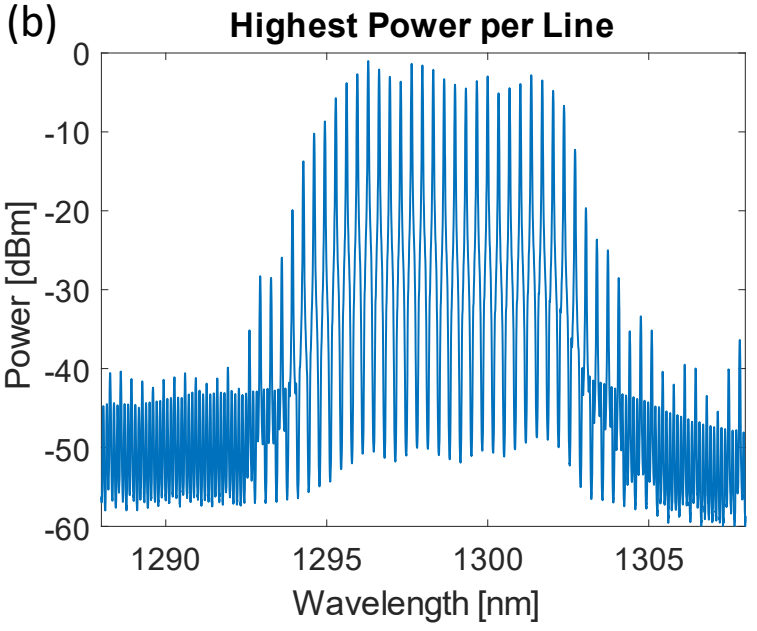

(d)

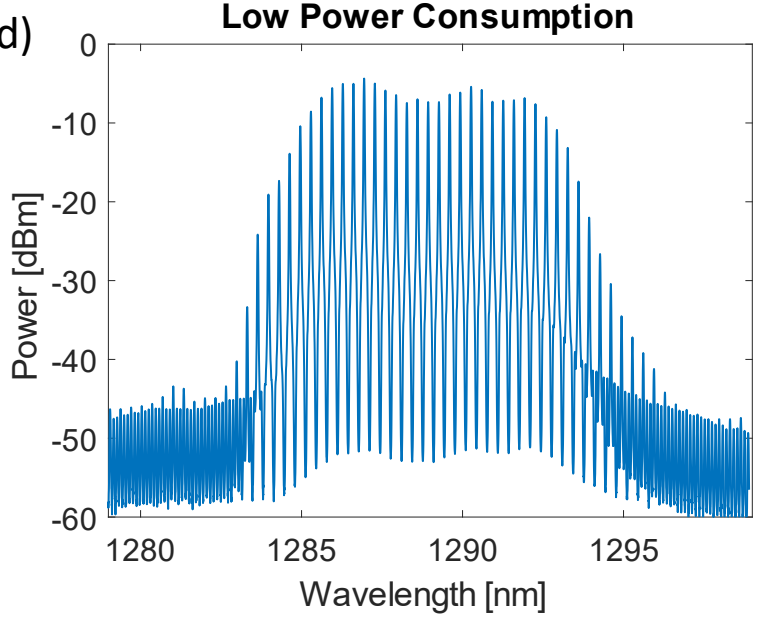

Figure 5. Optical spectra collected from the same device with a total saturable absorber length of $10 \%$ of the cavity. (a) Comb with the largest $6 \mathrm{~dB}$ bandwidth, 42 lines. (b) Comb with the highest power for 20 contiguous lines. (c) Comb with the highest efficiency of generating 20 contiguous lines. (d) Comb with low power consumption and high efficiency. 


\subsection{Comb Efficiency}

In order to analyze the efficiency of the different devices, the WPE of comb lines was analyzed. For a given comb, the comb line with the lowest power will also have the lowest WPE. Therefore, the PoLL values in Section 3.3 are divided by the electrical power consumption of the CPMLL for each state to calculate the WPE of the 20th channel (WPE20). The WPE20 for the devices from Figure 3 are shown in Figure $4 \mathrm{~d}-\mathrm{f}$, and the maximum and average WPE20 values are recorded in Table 1 . All WPE20 values are stated in dB and the PoLL used to calculate WPE20 includes a $2 \mathrm{~dB}$ chip-to-fiber coupling loss. The electrical power consumption was measured in a four-wire configuration. The WPE20 values closely track the PoLL values; however, states with lower drive current tend to have higher WPE20, which reflects the trend of the plots in Figure $3 \mathrm{~b}$. The $6 \%$ and $10 \%$ devices can both achieve similar WPE20, despite the $6 \%$ device having a shorter SA and a higher overall WPE for the same SA bias. The $6 \%$ device requires higher reverse bias to achieve a more efficient (20-line) comb, which degrades its WPE. In the 18\% device, the saturable loss is considerably higher, which reduces the average efficiency of comb generation and moves efficient states to higher drive currents and lower SA biases. This demonstrates that the bias values of efficient comb generation can be tuned by changing the SA length; however, $10 \%$ offers the most efficient comb and the largest area of efficient combs. The state with the highest WPE is shown in Figure $5 \mathrm{c}$ from the 10\% device at a drive current of $140 \mathrm{~mA}$ and an SA bias of $1.8 \mathrm{~V}$. This state consumes $214 \mathrm{~mW}$ of electrical power and produces $20 \mathrm{comb}$ lines with powers ranging from -5.62 to $-4.35 \mathrm{dBm}$. Another comb from the $10 \%$ device is shown in Figure $5 \mathrm{~d}$ with a lower power consumption, and a $0.12 \mathrm{~dB}$ reduction in efficiency from the state shown in Figure 5c. The comb is produced with $144 \mathrm{~mW}$ of electrical power, at an SA bias of $1.8 \mathrm{~V}$ and a drive current of $100 \mathrm{~mA}$, and supplies 20 lines ranging from -7.46 to $-4.37 \mathrm{~dB}$. This demonstrates the ability of CPMLLs to produce efficient combs at a range of output powers.

\section{Conclusions}

We have reported the design, fabrication, and performance of QD CPMLLs that operate in the O-band. The devices were extensively characterized and they demonstrated exceptionally high comb-generation efficiency. We showed lasers with the ability to generate 20-40 channels, spaced at $60 \mathrm{GHz}$. The analysis demonstrates significant progress towards the implementation of comb sources in low power consumption, small footprint DWDM transmitters. Furthermore, these devices are compact, simple to operate, and robust, simplifying transceiver design.

Author Contributions: Conceptualization, S.L. and J.B.; methodology, M.D. and S.L.; software, M.D.; validation, M.D.; formal analysis, M.D.; investigation, M.D.; resources, J.B. and M.J.K.; data curation, M.D.; writing—original draft preparation, M.D.; writing—review and editing, M.D. and J.B.; visualization, M.D.; supervision, J.B.; project administration, J.B.; funding acquisition, J.B. All authors have read and agreed to the published version of the manuscript.

Funding: This research was funded by the DARPA PIPES program HR0011-19-C-0083 and DOE ARPA-E ULTRALIT, contract number DE-AR0001039.

Institutional Review Board Statement: Not applicable.

Informed Consent Statement: Not applicable.

Data Availability Statement: Available on Zenodo (https://zenodo.org/record/5997357\#.YgE4 npbMKUk accessed on 4 November 2021).

Acknowledgments: The authors would like to thank Gordon Keeler, Justin Norman, and Alan Liu for fruitful conversations.

Conflicts of Interest: J.B. is cofounder of Quintessent, a silicon photonics company making mode locked lasers. 


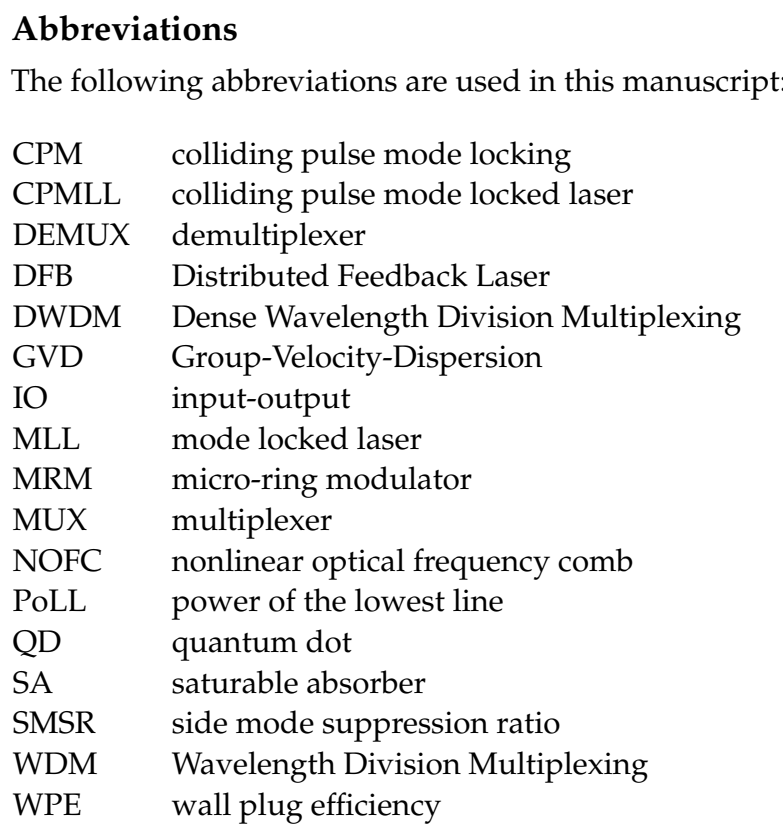

\section{References}

1. Cisco Visual Networking. The Zettabyte Era-Trends and Analysis. Cisco White Paper. 2018. Available online: https://www.cisco. $\mathrm{com} / \mathrm{c} / \mathrm{en} / \mathrm{us} /$ solutions/collateral/service-provider/visualnetworking-index-vni/vni-hyperconnectivity-wp.html (accessed on 4 November 2021).

2. Ghiasi, A. Large data centers interconnect bottlenecks. Opt. Express 2015, 23, 2085. [CrossRef] [PubMed]

3. Cheng, Q.; Bahadori, M.; Glick, M.; Rumley, S.; Bergman, K. Recent advances in optical technologies for data centers: A review. Optica 2018, 5, 1354. [CrossRef]

4. Margalit, N.; Xiang, C.; Bowers, S.M.; Bjorlin, A.; Blum, R.; Bowers, J.E. Perspective on the future of silicon photonics and electronics. Appl. Phys. Lett. 2021, 118, 220501. [CrossRef]

5. Komljenovic, T.; Huang, D.; Pintus, P.; Tran, M.A.; Davenport, M.L.; Bowers, J.E. Photonic Integrated Circuits Using Heterogeneous Integration on Silicon. Proc. IEEE 2018, 106, 2246-2257. [CrossRef]

6. Malik, A.; Liu, S.; Timurdogan, E.; Harrington, M.; Netherton, A.; Saeidi, M.; Blumenthal, D.J.; Theogarajan, L.; Watts, M.; Bowers, J.E. Low power consumption silicon photonics data center interconnects enabled by a parallel architecture. In Optical Fiber Communication Conference; Optical Society of America: San Diego, CA, USA, 2021; p. W6A.3.

7. Zheng, X.; Chang, E.; Shubin, I.; Li, G.; Luo, Y.; Yao, J.; Thacker, H.; Lee, J.H.; Lexau, J.; Liu, F.; et al. A 33 mW 100 Gbps CMOS silicon photonic WDM transmitter using off-chip laser sources. In Proceedings of the 2013 IEEE Optical Fiber Communication Conference and Exposition and the National Fiber Optic Engineers Conference (OFC/NFOEC), Anaheim, CA, USA, 17-21 March 2013; p. PDP5C.9.

8. Chang, L.; Liu, S.; Bowers, J.E. Integrated Optical Frequency Comb Technologies. Nat. Photonics 2022, 16, 95-108. [CrossRef]

9. Galdino, L.; Edwards, A.; Yi, W.; Sillekens, E.; Wakayama, Y.; Gerard, T.; Pelouch, W.S.; Barnes, S.; Tsuritani, T.; Killey, R.I.; et al. Optical Fibre Capacity Optimisation via Continuous Bandwidth Amplification and Geometric Shaping. IEEE Photonics Technol. Lett. 2020, 32, 1021-1024. [CrossRef]

10. Distributed Feedback Lasers. Available online: https://nanoplus.com/en/dfb-lasers/ (accessed on 4 November 2021).

11. Zheng, X.; Lin, S.; Luo, Y.; Yao, J.; Li, G.; Djordjevic, S.S.; Lee, J.H.; Thacker, H.D.; Shubin, I.; Raj, K.; et al. Efficient WDM Laser Sources Towards Terabyte/s Silicon Photonic Interconnects. J. Light. Technol. 2013, 31, 4142-4154. [CrossRef]

12. Marin-Palomo, P.; Kemal, J.N.; Karpov, M.; Kordts, A.; Pfeifle, J.; Pfeiffer, M.H.P.; Trocha, P.; Wolf, S.; Brasch, V.; Anderson, M.H.; et al. Microresonator-based solitons for massively parallel coherent optical communications. Nature 2017, 546, 274-279. [CrossRef] [PubMed]

13. Pavlov, N.G.; Koptyaev, S.; Lihachev, G.V.; Voloshin, A.S.; Gorodnitskiy, A.S.; Ryabko, M.V.; Polonsky, S.V.; Gorodetsky, M.L. Narrow-linewidth lasing and soliton Kerr microcombs with ordinary laser diodes. Nat. Photonics 2018, 12, 694-698. [CrossRef]

14. Pfeiffer, M.H.P.; Herkommer, C.; Liu, J.; Guo, H.; Karpov, M.; Lucas, E.; Zervas, M.; Kippenberg, T.J. Octave-spanning dissipative Kerr soliton frequency combs in Si_3N_4 microresonators. Optica 2017, 4, 684. [CrossRef]

15. Harrington, M.W.; Brodnik, G.M.; Briles, T.C.; Stone, J.R.; Streater, R.H.; Papp, S.B.; Blumenthal, D.J. Kerr Soliton Microcomb Pumped by an Integrated SBS Laser for Ultra-Low Linewidth WDM Sources. In Optical Fiber Communication Conference (OFC) 2020; OSA: San Diego, CA, USA, 2020; p. T4G.6. [CrossRef]

16. Bao, C.; Zhang, L.; Matsko, A.; Yan, Y.; Zhao, Z.; Xie, G.; Agarwal, A.M.; Kimerling, L.C.; Michel, J.; Maleki, L.; et al. Nonlinear conversion efficiency in Kerr frequency comb generation. Opt. Lett. 2014, 39, 6126. [CrossRef] 
17. Kim, B.Y.; Okawachi, Y.; Jang, J.K.; Yu, M.; Ji, X.; Zhao, Y.; Joshi, C.; Lipson, M.; Gaeta, A.L. Turn-key, high-efficiency Kerr comb source. Opt. Lett. 2019, 44, 4475. [CrossRef] [PubMed]

18. Fülöp, A.; Mazur, M.; Lorences-Riesgo, A.; Helgason, Ó.B.; Wang, P.H.; Xuan, Y.; Leaird, D.E.; Qi, M.; Andrekson, P.A.; Weiner, A.M.; et al. High-order coherent communications using mode-locked dark-pulse Kerr combs from microresonators. Nat. Commun. 2018, 9, 1598. [CrossRef] [PubMed]

19. Xiang, C.; Liu, J.; Guo, J.; Chang, L.; Wang, R.N.; Weng, W.; Peters, J.; Xie, W.; Zhang, Z.; Riemensberger, J.; et al. Laser soliton microcombs heterogeneously integrated on silicon. Science 2021, 373, 99-103. [CrossRef]

20. Hu, H.; Da Ros, F.; Pu, M.; Ye, F.; Ingerslev, K.; Porto da Silva, E.; Nooruzzaman, M.; Amma, Y.; Sasaki, Y.; Mizuno, T.; et al Single-source chip-based frequency comb enabling extreme parallel data transmission. Nat. Photonics 2018, 12, 469-473. [CrossRef]

21. Delfyett, P.; Hartman, D.; Ahmad, S. Optical clock distribution using a mode-locked semiconductor laser diode system. J. Light. Technol. 1991, 9, 1646-1649. [CrossRef]

22. Wada, O. Femtosecond all-optical devices for ultrafast communication and signal processing. New J. Phys. 2004, 6, 183. [CrossRef]

23. Liu, S.; Wu, X.; Jung, D.; Norman, J.C.; Kennedy, M.J.; Tsang, H.K.; Gossard, A.C.; Bowers, J.E. High-channel-count 20 GHz passively mode-locked quantum dot laser directly grown on Si with 41 Tbit/s transmission capacity. Optica 2019, 6, 128. [CrossRef]

24. Asada, M.; Miyamoto, Y.; Suematsu, Y. Gain and the threshold of three-dimensional quantum-box lasers. IEEE J. Quantum Electron. 1986, 22, 1915-1921. [CrossRef]

25. Liu, G.; Stintz, A.; Li, H.; Malloy, K.; Lester, L. Extremely low room-temperature threshold current density diode lasers using InAs dots in In0.15Ga0.85As quantum well. Electron. Lett. 1999, 35, 1163. [CrossRef]

26. Chow, W.W.; Zhang, Z.; Norman, J.C.; Liu, S.; Bowers, J.E. On quantum-dot lasing at gain peak with linewidth enhancement factor $\alpha_{\mathrm{H}}=0$. APL Photonics 2020, 5, 026101. [CrossRef]

27. Akiyama, T.; Ekawa, M.; Sugawara, M.; Kawaguchi, K.; Sudo, H.; Kuramata, A.; Ebe, H.; Arakawa, Y. An ultrawide-band semiconductor optical amplifier having an extremely high penalty-free output power of $23 \mathrm{dBm}$ achieved with quantum dots. IEEE Photonics Technol. Lett. 2005, 17, 1614-1616. [CrossRef]

28. Arakawa, Y.; Sakaki, H. Multidimensional quantum well laser and temperature dependence of its threshold current. Appl. Phys. Lett. 1982, 40, 939-941. [CrossRef]

29. Vallaitis, T.; Koos, C.; Bonk, R.; Freude, W.; Laemmlin, M.; Meuer, C.; Bimberg, D.; Leuthold, J. Slow and fast dynamics of gain and phase in a quantum dot semiconductor optical amplifier. Opt. Express 2008, 16, 170. [CrossRef] [PubMed]

30. Thompson, M.; Marinelli, C.; Zhao, X.; Sellin, R.; Penty, R.; White, I.; Kaiander, I.; Bimberg, D.; Kang, D.J.; Blamire, M. Colliding-pulse modelocked quantum dot lasers. Electron. Lett. 2005, 41, 248. [CrossRef]

31. Liu, S.; Jung, D.; Norman, J.C.; Kennedy, M.J.; Gossard, A.C.; Bowers, J.E. 490 fs pulse generation from passively mode-locked single section quantum dot laser directly grown on on-axis GaP/Si. Electron. Lett. 2018, 54, 432-433. [CrossRef]

32. Wan, Y.; Jung, D.; Norman, J.; Feng, K.; Dagli, A.; Gossard, A.C.; Bowers, J.E. Quadruple reduction of threshold current density for micro-ring quantum dot lasers epitaxially grown on (001) Si. In Conference on Lasers and Electro-Optics; OSA: San Jose, CA, USA, 2018; p. SW3Q.3. [CrossRef] 